\title{
Kernos
}

Revue internationale et pluridisciplinaire de religion grecque antique

18 | 2005

Varia

\section{Retour à la terre : fin de la Geste d'Érechthée}

\section{Sonia Darthou}

Édition électronique

DOI : $10.4000 /$ kernos.900

ISSN : 2034-7871

\section{Éditeur}

Centre international d'étude de la religion grecque antique

\section{Édition imprimée}

Date de publication : 1 janvier 2005

Pagination : 69-83

ISSN : 0776-3824

Référence électronique

Sonia Darthou, «Retour à la terre : fin de la Geste d'Érechthée », Kernos [En ligne], 18 | 2005, mis en ligne le 16 juin 2011, consulté le 02 mai 2019. URL : http://journals.openedition.org/kernos/900; DOI : 10.4000/kernos.900 


\section{Retour à la terre : fin de la Geste d'Érechthée}

Résumé : En terre d'Athènes, on ne trouve pas un fondateur, mais des autochtones, qui multiplient la figure inaugurale du fondateur sans pour autant l'incarner complètement. C'est Érechthée qui, par sa mort inédite, va clôturer la longue chaîne des opérations de fondation. Caché dans la terre civique sous les coups du trident vengeur de Poséidon, sa mort n'est ni un crime, ni une «belle mort », mais bien une mort fondatrice. La mort d'Érechthée donne en effet lieu à un partenariat de choix pour ces Athéniens en mal d'ancrage : Érechthée, le roi autochtone et Poséidon, le dieu à l'assise solide et aux fondations inébranlables, qui seront désormais associés dans le culte sous le nom de Poséidon-Érechthée ou Érechthée-Poséidon. Grâce à ce partenariat fondateur, la cité autochtone a réussi le pari de son auto-fondation. Car, à travers Érechthée, c'est tous les Athéniens que Poséidon enracine sur leur propre terre.

Abstract: Return to earth: the end of the story of Erechtheus. There is not one founder of Athens but many autochtones repeating the inaugural figure of the founder without nonetheless incarnating it fully. It is the particular death of Erechtheus that ends the long founding operations. Hidden in the civic earth, his death under Poseidon's revengeful trident is neither a crime nor a "beautiful death" but a founding death. Indeed, the death of Erechtheus gives birth to a founding partnership of choice for those Athenians in search of their roots: Erechtheus, the autochtonous king, and Poseidon, the god with a solid basis and unmovable foundations, would become associated in the cult as Poseidon-Erechtheus or Erechtheus-Poseidon. Because of this founding partnership, the autochtonous city succeeded in the challenge of self-foundation. Through Erechtheus, it was all Athenians that Poseidon could root in their own land.

Malgré ses glorieuses origines encensées par les orateurs, Athènes l'autochtone est toujours en quête de sa propre fondation. N'ayant pas été fondée sur le mode de la colonisation mais «autofondée ", elle cherche sans cesse à renouer des liens avec la terre qui l'a générée pour assurer toujours plus son ancrage. Son autochthonie initiale s'avère en effet bien fragile dans une terre grecque qui valorise de complexes opérations de fondation : tracer l'enceinte, découper le territoire, se donner un fondateur architecte et archégète'. Or, dans la cité, le processus de fondation se décline moins en opérations « horizontales» qui tendent à défricher/délimiter le nouveau territoire qu'en prise de possession «verticale » du sol. En terre d'Athènes, en effet, on ne trouve pas un fondateur, mais des autochtones qui, par leurs singulières naissances,

1 Voir M. Detienne, Apollon le couteau à la main, Paris, 1998, p. 85-134. Sur le vocabulaire de la fondation/colonisation, voir M. CASEVITZ, Le Vocabulaire de la colonisation en grec ancien.

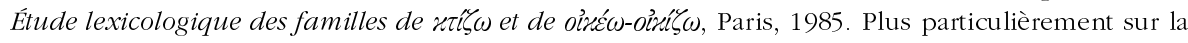
figure de l'archégète, voir p. 245-250 et sur les fondateurs de colonies voir I. MALKIN, Religion and Colonisation in Ancient Greece, Leiden et al., 1987, p. 17-91. 
multiplient la figure inaugurale du fondateur, symbolisent les racines de la cité et garantissent ainsi l'autofondation de la polis.

La longue liste de ces autochtones ${ }^{2}$ où se succèdent Kékrops - le premier roi - et Érichthonios - le premier Athénien -, aboutit à Érechthée qui, s'il s'approche de la figure du fondateur ${ }^{3}$, n'effectue pourtant aucun geste de territorialisation. Il naît, il nomme, mais il ne trace pas. En fait, son acte «fondateur » est, plus que de naître, de mourir sous les coups de Poséidon qui le fait retourner dans le chthôn générateur. Les textes, en effet, ne s'attardent pas à raconter la naissance d'Érechthée: germer de la terre est l'apanage d'Érichthonios. Pour le roi autochtone, c'est sa mort qui est le point culminant de sa geste. Et le terme ultime des fondations d'Athènes.

La mort particulière d'Érechthée donne en effet naissance à une alliance fondamentale pour les bonnes fondations de la cité : celle du roi autochtone et du Poséidon à l'Assise sûre que les Grecs honorent comme 'Stable'

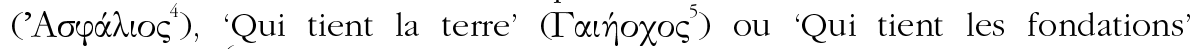
$(\Theta \varepsilon \mu \varepsilon \lambda \iota 0 \tilde{\chi} \chi O c)^{6}$. Nous avons déjà analysé les compétences terriennes de Poséidon dans notre travail de thèse ${ }^{7}$ : tenir/soutenir le sol, affermir le socle, assurer les assises, enraciner et soutenir les fondations. À l'issue de cet épisode, Poséidon prend la place du fondateur au côté d'un Érechthée qui, ancré dans la terre sous les coups de son trident, prend vraiment sa dimension d'autochtone.

\section{Cacher Érechthée dans la terre civique}

Toute l'histoire se joue pendant la fameuse querelle qui oppose Athéna et Poséidon, les deux prétendants au titre de Poliade. Une initiale querelle (eris) sous le roi hybride Kékrops ${ }^{8}$ qui devient un combat (polemos) sous le règne d'Érechthée. Dans cette version privilégiée par Euripide et les orateurs attiques ${ }^{9}$, deux camps se dessinent: d'un côté Athènes, la déesse et le roi

2 Voir P. Brulé, «La Liste des premiers rois d'Athènes dans la Bibliothèque du PseudoApollodore : histoire, politique et parenté », Poikilia (1996), p. 37-53.

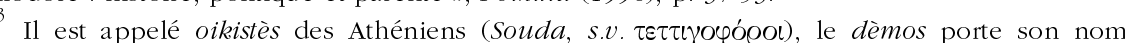
(Hom., Il. II, 547), les Athéniens sont appelés Érechthéides (Ar., Cav., 1015; Soph., Aj., 202 et Pind., Isthm. II, 19) et il donne à la cité son nom définitif (Hdt., VIII, 44).

4 Paus., VII, 21, 7 indique qu'on lui donne partout cette épiclèse.

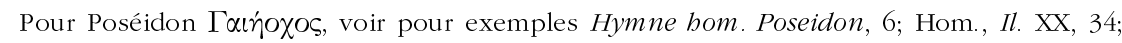
Od., I, 68; III, 55; VIII, 322, 350; IX, 528 sq.

En Attique, voir K. CLINTON, "The Sacred Officials of The Eleusinians Mysteries », in TAPhA 64.3 (1974), p. 50-52, 17 et plus généralement schol. Hom., Il. XXI, 447b à propos de la construction du mur de Troie.

7 «Poséidon en terre d'Athènes : un dieu entre séisme et fondation », EPHE juin 2000.

8 Pour exemples, Apoll., Bibl. III, 14, 1; Plut., Quaest. Conv., 9, 6 (Mor., 741) et Hdt., VIII, 55.

9 Eur., Er., que nous citons dans l'édition de P. CARRARA, " Euripide. Eretteo », in Papyrologica Florentina vol. III (1977); pour les orateurs, voir Lycurgue, Leocr., 98-100; Isocrate, Pan., 66-67 et Démosthène, Epit., 8. 
Érechthée, et de l'autre Éleusis, Poséidon et son fils Eumolpe, allié aux terribles Thraces.

Dès l'engagement du polemos, Athènes consulte les dieux qui lui intiment de sacrifier la(les) fille(s) du roi pour remporter la victoire ${ }^{10}$ : elles sont tour à tour dans nos sources anonymes ${ }^{11}$, prénommées Chthonia et/ou AglaurosAgraulos $^{12}$. Plusieurs versions se font aussi écho : selon Philochore, l'oracle réclame une victime nommée Agraulos qui, pour sauver la cité, choisit de se donner la mort en se précipitant du haut des murailles ${ }^{13}$. Hygin ${ }^{14}$ indique lui que Poséidon, endeuillé et voulant empêcher qu'Érechthée ne se réjouisse de la mort d'Eumolpe, demande qu'une de ses filles soit sacrifiée (immolaretur). C'est Chthonia qui est désignée et ses sours la suivent fidèlement dans la mort. Quant à Apollodore, il rapporte que le roi égorge sa fille cadette

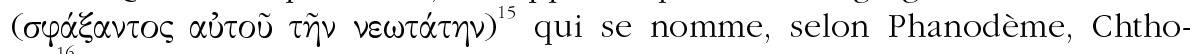
$\mathrm{nia}^{16}$.

En dépit de ces divergences, l'oracle une fois rendu, les deux factions rivales peuvent s'affronter : si Érechthée tue Eumolpe, le fils de Poséidon, chef des Éleusiniens ${ }^{17}$, le camp athénien doit lui aussi subir le deuil de son roi, avalé dans une faille de la terre ouverte par un Poséidon vengeur.

${ }^{10}$ Lyc., Leocr., 99; Démarate, FGrHist. 42 F 4 Jacoby; Arist., Or., 87 et Eur., Ion, 277. Selon Hygin, Fab., 46, c'est Poséidon qui demande le sacrifice. Pour les différentes versions, voir H. VAN LoOY, «L'Érechthée d'Euripide », in Mélanges en hommage à Marie Delcourt, Paris, 1980, p. 116-117. Sur le sacrifice, voir J.C. KAMERBEEK, «En relisant les fragments de l'Érechthée d'Euripide », in H. HOFFMANN \& A. HARDER (éds), Fragmenta Dramatica, Göttingen, 1991, p. 111116; J. Wilkins, "The State and The Individual: Euripides' Plays of Voluntary Self-Sacrifice », in A. Powell (éd.), Euripides, Women, and Sexuality, London / New York, 1990, p. 177-194.

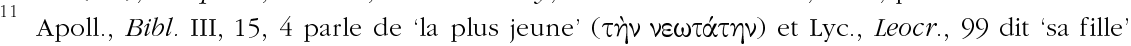
( $\tau \dot{\eta} \nu \theta 0 \gamma \alpha \tau \hat{\varepsilon} Q \alpha)$

Sur ce double nom, voir P. Brulé, La Fille d'Athènes, Paris, 1987, p. 28-29 et M. Detienne, «La Force des femmes », in G. SISSA \& M. DetiEnne, La Vie quotidienne des dieux grecs, Paris, 1989 , p. 245.

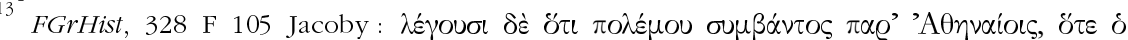

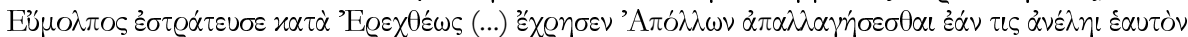

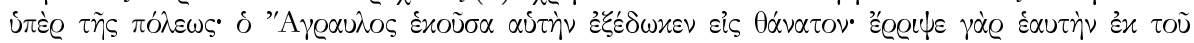
$\tau \varepsilon i \xi o u c$.

Fab., 46 et 238 qui rapportent une tradition similaire.

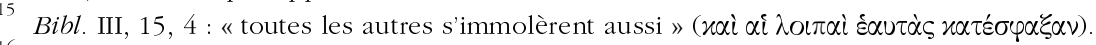

${ }^{16}$ FGrHist, 325 F 4 Jacoby. Démarate (42 F 4 Jacoby) choisit de mentionner le sacrifice de

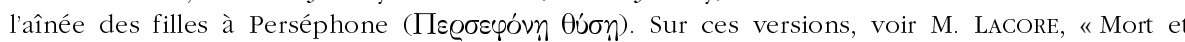
divinisation des filles du roi d'Athènes dans l'Érechthée d'Euripide », in Kentron 11,2-12,1 (19951996), p. $91 s q$.

Apoll., Bibl. III, 15, 4 : «dans la bataille qui suivit le sacrifice, Érechthée tua Eumolpos »

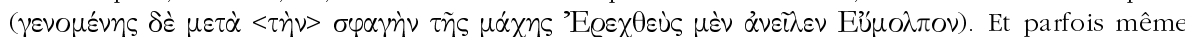
d'autres descendants du dieu : une scholie (ad Eur., Phen., 854)) ajoute que deux autres fils de Poséidon, Phorbas et Immarados, sont aussi tués par Érechthée (Eư $\mu \circ \lambda \pi O \varsigma$ (...) ò $\pi$ ò $\varepsilon \mu \tilde{\omega} \nu \tau \dot{\alpha} \varsigma$

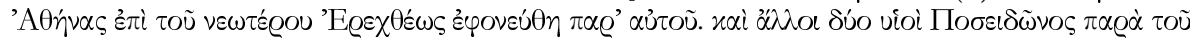

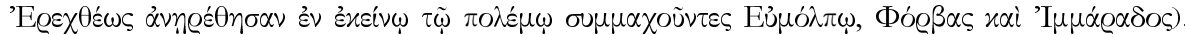

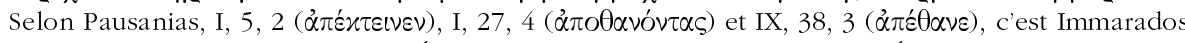
et non Eumolpos qui fut tué par Érechthée. C'est en tout cas le combat Érechthée/Eumolpos qui est repris par Myron sur l'Acropole (I, 5, 2 et I, 27, 4). 
Quelle que soit la version privilégiée, la mort d'Érechthée contraste par sa non-violence avec le sang féminin qui coule sur l'autel de la patrie : car, en face de $\sigma \varphi \alpha^{\prime} \zeta \omega^{18}$ et de $\theta u ́ \omega^{19}$, nous trouvons en effet pour qualifier la mort du

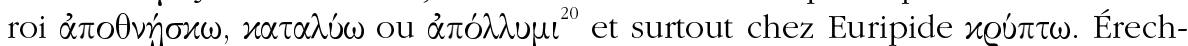
thée a donc été enfoui (caché) sous (dans) la terre ( $\alpha_{\alpha} \dot{\alpha} \chi \theta$ ovò $\left.\varkappa Q u ́ \psi \alpha \varsigma\right)^{21}$. Ce verbe kruptô a la signification d'« envelopper pour cacher», parfois même avec une nuance de protection ${ }^{22}$, ou de "cacher, dissimuler », de soustraire aux regards ${ }^{24}$, de recouvrir aussi d'une manière plus métaphorique ${ }^{25}$. Dans toutes ces occurrences, cacher ou enlever à la vue, on doit remarquer que kruptô ne recèle pas de violence: il s'agit plus d'enfouir. Mais ce verbe contient aussi indéniablement une dimension funéraire ${ }^{26}$. Si l'on continue avec le même auteur Euripide, on retrouve en effet souvent kruptô pour décrire la mise en terre des défunts ${ }^{27}$, et être caché / enfoui dans le sol devient une des métaphores de la mise en terre ${ }^{28}$. C'est d'ailleurs le champ lexical de kruptô $^{29}$ qui est utilisé pour dire l'obligation rituelle d'enfouir dans la terre ${ }^{30}$; et c'est

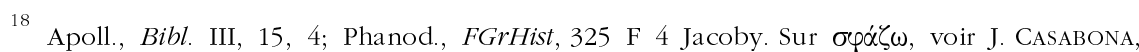
Recherches sur le vocabulaire des sacrifices en grec des origines à la fin de l'époque classique, Ophrys, 1966, p. 155-167 et J. RUDHARDT, Notions fondamentales de la pensée religieuse et actes constitutifs du culte dans la Grèce classique, Paris, 1992 [1958], p. 272-281.

19 Démar., FGrHist 42 F 4 Jacoby; Eur., Ion, 277-78; Lyc., Leocr., 100. Sur Oúw, voir CASABONA, o.c. (n. 18), p. 69-94

Pour Pausanias (I, 38, 3) le roi meurt ( $\alpha \dot{\varepsilon} \theta \alpha \nu \varepsilon)$ au cours de la bataille; Apollodore (Bibl. III, 15, 5) parle de destruction : «Poséidon détruisit Érechthée avec sa maison » ( $\alpha \alpha \tau \lambda \dot{\sigma} \sigma \alpha \nu \tau O \varsigma)$;

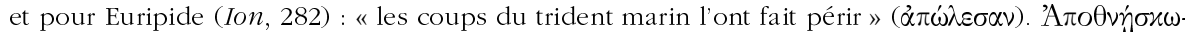
mourir, $x \alpha \tau \alpha \lambda \hat{\omega} \omega$-détruire et $\alpha \dot{\alpha} \hat{\lambda} \lambda \cup \mu t$-perdre, faire périr, aucun de ces verbes n'induit le meurtre.

${ }^{21}$ Deux pièces d'Euripide se font l'écho de cette version. Dans l'Érechthée (18, 59-60), Praxithéa à Poséidon : " cela ne t'a-t-il pas satisfait pleinement d'avoir englouti Érechthée dans le

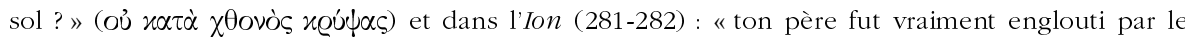

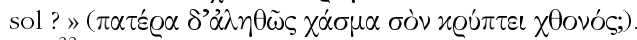

${ }^{22}$ Notamment quand on parle d'armure qui protège le combattant (Eur., Phen., 1242 sq.).

23 P. Chantraine, Dictionnaire étymologique de la langue grecque. Histoire des mots, Paris,

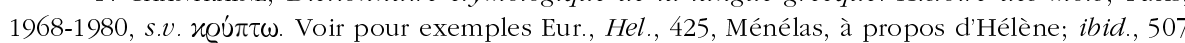
et Eur., I.T., 119 pour la notion de cachette.

24 Soph., $A j$., 658. Voir aussi Ar., Thesm., 600 pour ce sens de cacher à la vue.

25 On dit qu'une armée ennemie a recouvert Troie selon Eur., Hec., 905-907.

26 Soph., Ant., 21-25 : "Pour Étéocle (...) il l'a fait ensevelir d'une manière qui lui vaille le

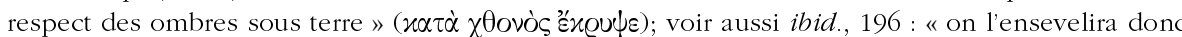

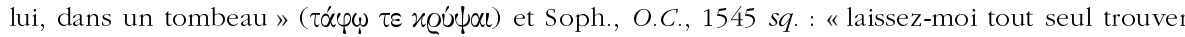

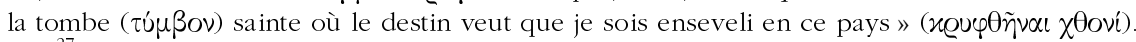

${ }^{27}$ Eur., Hel., 1222-1223 : «A-t-il abandonné ton mari sans tombe ou l'ont-ils enfoui sous la terre ?" (

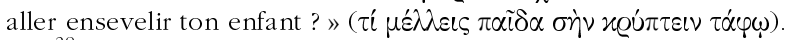

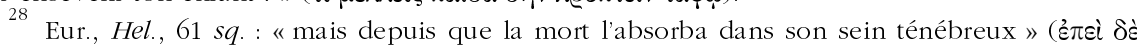

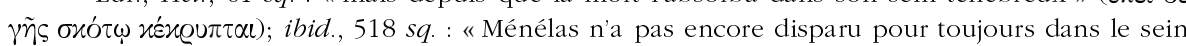

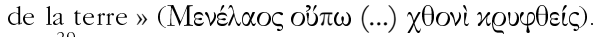

29 Lois XII, 958d-e : "aucune tombe $(\theta \dot{n} \varkappa \alpha \varsigma)$ ne sera permise nulle part en terre labourable (...); là seulement où la nature du sol n'est bonne qu'à cette fin, à recevoir et cacher les corps des

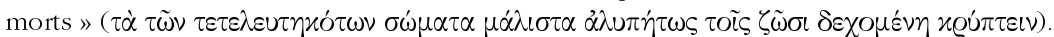

Selon Paus., I, 32, 5 : «d'une manière générale, c'est une règle sainte (óбıov) que de 
aussi à partir du moment où les corps ont été " cachés-confiés » à la terre que l'oraison funèbre de Périclès peut commencer ${ }^{31}$.

Néanmoins, si Érechthée est enfoui dans la terre attique sous les coups de Poséidon, le lieu de son engloutissement n'est pas pour autant sa tombe ${ }^{32}$ : si Euripide reconnaît qu'Érechthée est bien sous terre ${ }^{33}$, il ne mentionne pas de tombe pour le roi autochtone mais bien un sèkos ${ }^{34}$. On a remarqué que si le verbe kruptô recèle une dimension funéraire, il ne suffit pas seul à dire la mise à la tombe, plus précisément concerné par le fait de cacher et d'ensevelir dans le sous-sol. Et c'est bien ce qui nous importe ici car, pour Érechthée, le chthôn s'est substitué au taphos ou au tumbos ${ }^{35}$.

La tombe est, elle, réservée à Érichthonios qui, selon Apollodore ${ }^{36}$, a été enseveli dans le temenos d'Athéna. Mais, pour Érechthée, il n’y a ni sèma ni commémoration funéraire. Son engloutissement est avant tout le point de départ d'un double culte qui valorise la nouvelle association entre Poséidon et Érechthée, entre l'« enfouisseur » et sa «victime », qui vont désormais partager un autel dans l'Érechthéion.

Ce geste de cacher dans une brisure du sol (un $\chi \alpha \dot{\alpha} \sigma \mu \alpha$ ) rappelle bien sûr les compétences hautement telluriques de Poséidon qui secoue et déchire la terre les jours de colère ${ }^{37}$. Mais si le chasma peut être signe de désordre et de violence, on a surtout ici à en retenir son aspect d'ouverture $(\sigma x i \sigma \mu \alpha)$, voire de bouche $(\sigma \tau o ́ \mu \alpha)$ à la suite d'Hésychius ${ }^{38}$ : Poséidon, en fendant la terre,

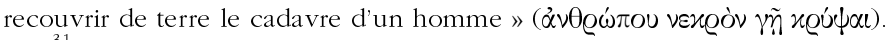

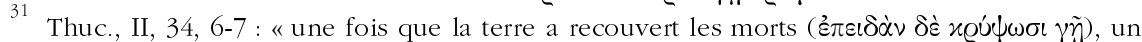
homme choisi par la cité (...) prononce en leur honneur un éloge approprié (...). Ainsi ont lieu les funérailles » ("$\Omega \delta \varepsilon \mu \dot{\varepsilon} \nu \theta \dot{\alpha} \pi \tau O \nu \sigma เ v)$.

32 Comme G.W. ElDERKIN, "The Cults of the Erechtheion », Hesperia 10 (1941), p. 113 : "Poseidon's trident drove Érechtheus into the earth. The marks of it were the site of his tomb. » K.H. LEE, Euripides. Ion, Warminster, 1997, p. 191 à propos des vers 281-282 écrit lui (mais sans références) que l'endroit où Poséidon a ouvert la roche pour faire surgir la thalassa "was the supposed grave of Erechtheus ». Thèse soutenue également par M. CHRISTOPOULOS,

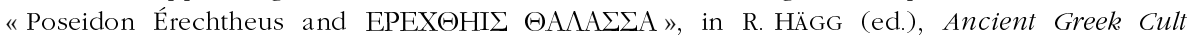
Practice from The Epigraphical Evidence, Stockholm, 1994, p. 128.

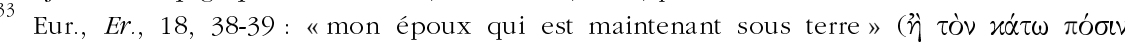

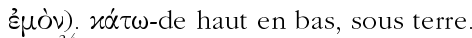

34 Eur., Er., 18, 90.

35 Voir Soph., Ant., 196 et id., O.C., 1545 (cité supra note 26).

Apoll., Bibl. III, 14, 7 : «À sa mort, Érichthonios fut enseveli dans le sanctuaire même

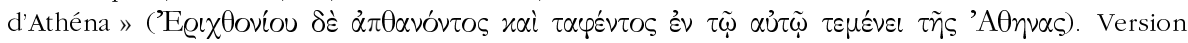
similaire chez Clément d'Alexandrie, qui énumère les héros ensevelis dans les sanctuaires,

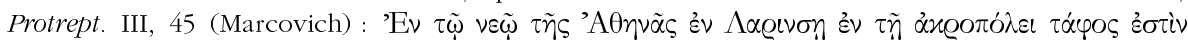

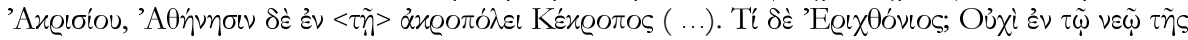

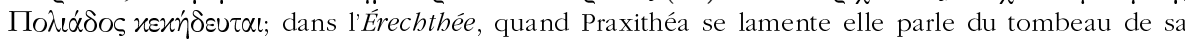
fille (taphos, 18.37) alors que Érechthée est « sous terre » (18, 39, voir supra note 33).

Comme Sparte (Plut., Cim., 16, 4), Sipyle (Paus., VII, 24, 13) et Bourra (Strabon, I, 3, 18) qui disparaissent dans un chasma.

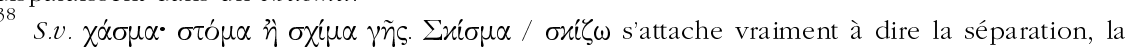
déchirure. 
ouvre $^{39}$ la porte du chthôn pour l'autochtone Érechthée et permet ainsi son retour dans sa terre de naissance. On sait par ailleurs combien les Athéniens aiment mettre en écho leur naissance autochtone et leur ultime mise en terre $^{40}$. Érechthée en est le paradigme. Tout le vocabulaire attaché à la mort de l'autochtone va ainsi s'éloigner du meurtre pour privilégier l'ancrage.

\section{Traces de sang sur l'Acropole?}

La mort d'Érechthée ne relève pas en effet du sacrifice, du meurtre ou de la blessure au flanc d'un combattant, car la question du sang est évacuée. Si Poséidon est appelé meurtrier ${ }^{41}$, le sang d'Érechthée ne coule pas pour la cité $^{42}$. D'ailleurs, ce terme de ktantas ne semble pas croiser la route du meurtre sanglant qui se dit phonos ${ }^{43}$. P. Chantraine ${ }^{44}$ indique en effet que $x \tau \varepsilon i v \omega$ ne comporte pas étymologiquement la signification de tuer, et que $\theta$ cív $\omega^{45}$ exprime plus la notion de «frapper».

On retrouve ailleurs ce souci de gommer le sang de la mort, toujours sous le signe de l'engloutissement. Car Érechthée n'est pas le seul à être avalé par un chasma; d'autre corps, bien différents certes, sont eux aussi engloutis dans des failles ${ }^{46}$. Une analyse de J.-L. Durand ${ }^{47}$ a été pour nous comme une invite à regarder de plus près d'autres cadavres, et notamment ceux très particuliers de certains condamnés à mort ${ }^{48}$. L'usage veut en effet qu'à Athènes et dans d'autres localisations helléniques, on précipite dans des failles excentrées ${ }^{49}$ les traîtres et les sacrilèges $^{50}$ : on connaît le Barathron à Athènes ${ }^{51}$, le Kéadas à Sparte $^{52}$, le Kôs à Corinthe $\mathrm{e}^{53}$ ou les Phédriades ${ }^{54}$ à Delphes.

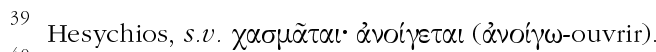

40 Platon, Men., 237b : «autochtones (...) nourris (...) par la terre maternelle qu'ils habitaient et qui ont permis à leurs fils de reposer morts ( $\tau \varepsilon \lambda \varepsilon u \tau \dot{\eta} \sigma \alpha \nu \tau \alpha \varsigma)$, aujourd'hui, dans les lieux familiers de celle qui les mit au monde. »

41 Eur., Er., 18, 93.

42 On doit pourtant admettre que dans l'Érechthée d'Euripide $(18,19)$ le messager dit qu'« il

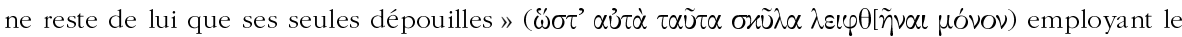
vocable $\sigma x u ́ \lambda \alpha$-dépouilles, qui induit la notion d'écorchement.

${ }^{43}$ Il faut tout de même mentionner que dans Eur., Er., 18, 40, les rafales sismiques sont dites

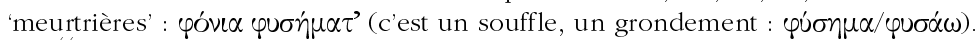

44 «Les Verbes signifiant 'tuer'», Die Sprache 1 (1949), p. 143-144.

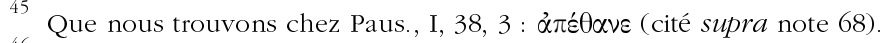

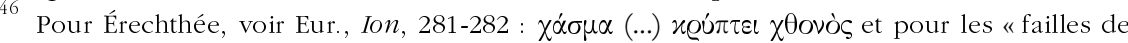

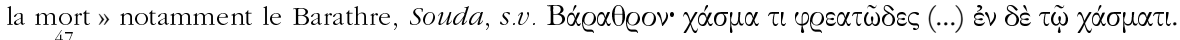

47 "La Mort, les morts et le reste», in M. DETIENnE \& M. CARTRY (éds), Destins de meurtriers. Systèmes de pensée en Afrique Noire, Paris, 1996, p. 49.

48 I. BARKAN, Capital Punishment in Ancient Athens, Chicago, 1936, a établi un des premiers dossiers sur la peine capitale, sans pourtant réellement analyser le mode de mise à mort.

49 À Athènes, le gouffre semble être hors des murs : pour les ambiguités de sa localisation, voir M. Halm-Tisserant, Réalités et imaginaires des supplices en Grèce ancienne, Paris, 1998, p. 152 et E. Cantarella, Il Suplizi capitali in Grecia e a Roma, Milan, 1991, p. 960 sq.

Ce châtiment ne semble pas aller au-delà du v viècle selon L. GERNET, «Le Droit pénal de la Grèce ancienne », in Du châtiment dans la cité, Supplices corporels et peine de mort dans le 
Cette peine capitale concerne les délits les plus importants, les crimes contre la cité $e^{55}$ et les crimes contre les dieux ${ }^{56}$ : deux catégories de criminels avec lesquels on semble prendre bien des précautions ${ }^{57}$. On évite en effet soigneusement que le sang ne coule dans la terre, on ne place pas d'arme non plus aux mains des bourreaux dans ce châtiment : la cité n'est pas meurtrière et ne veut pas prendre pas le risque de la souillure. Depuis L. Gernet ${ }^{58}$ et les premiers commentaires de Pausanias par J.G. Frazer ${ }^{59}$, on note, même si les sources font preuve de silence, que la mort par précipitation dans une faille évite de verser le sang ${ }^{60}$. Avalé dans le sol, le corps part intact dans la terre : pas d' $\alpha \tilde{i} \mu \alpha$ qui s'écoule au Barathre, pas d' $\alpha \tilde{i} \mu \alpha$ non plus sur l'Acropole pour Érechthée.

Mais on ne saurait trop mettre en parallèle les condamnés et le roi autochtone, même si ces corps sont «avalés » dans un gouffre qui permet d'occulter le sang de la blessure et de la mort. Leurs failles respectives montrent bien la divergence profonde qui les sépare. Le gouffre des condamnés ne se décline pas en effet sur le mode de chthôn ou de gè, mais bien de $\pi \dot{\tau} \tau \varrho \alpha^{61}$. D'un côté un chasma de pierre et de l'autre un chasma de terre. Tandis que les condamnés sont jetés sur la pierre ${ }^{62}$, Érechthée est lui « caché dans la terre ».

monde antique, Table ronde organisée par l'E.F.R. avec le concours du C.N.R.S., Rome 9-11 novembre 1982, Rome, 1984, p. 27 et CANTARELLA, o.c. (n. 49), p. 103.

${ }^{5}$ Xen., Hell. I, 7, 20; Hdt., VII, 133; Ar., Nub., 1449, Eq., 1362, Ran., 574, Pl., 431 et 1109. Puis Orygma au IV siècle qui recevait plus vraisemblablement des cadavres et non des condamnés vivants (Lyc., Leocr., 121). Voir L. GERNET, "Sur l'exécution capitale : à propos d'un ouvrage récent », in Droit et institutions en Grèce antique, Paris, 1982, p. 184 note 29

52 Paus., IV, 18, 4.

53 Et. de Byz., s.v. Kü $\varsigma$.

54 Plut., De sera nom. (Mor., 557a) ou Kéadas (Thuc., I, 134, 4).

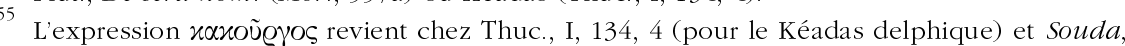
s.v. B $\alpha \varrho \alpha \theta \varrho o v$. On connaît le texte du décret de Cannônos (Xen., Hell. I, 7, 20) qui punit ceux qui ont enfreint la loi athénienne.

56 Halm-Tisserant, o.c. (n. 49), p. 156-157

7 Nos sources sont lapidaires. Dernièrement, HALM-TissERANT (o.c. (n. 49)) reprend le dossier mais n'analyse pas réellement ce mode de 'mise à mort' ni même la catégorie concernée des condamnés

58 O.c. (n. 50), p. 27.

59 Pausanias's Description of Greece, Volume III. Commentary of Books II-V, New York, 1965, ad IV, 18, 4, à propos du Kéadas il note «it was perhaps adopted to save the executioners from actually spilling the blood of their victims » et renvoie à W. ROBERTSON SMITH, Lectures on The Religion of The Semites: First Series, The Fundamental Institutions, Edinburg, 1889, p. 417. Cet auteur indique que " care was taken to slay the victim without bloodshed, or to make believe that it had killed itself».

60 DuRAND, o.c. (n. 47), p. 49 en renvoyant aussi aux recherches de ROBERTSON SMITH, o.c. (n. 59), p. 417

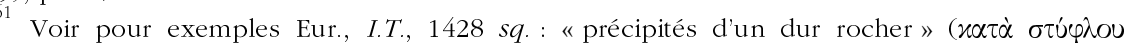
$\pi \varepsilon ́ \tau \varrho \alpha \varsigma$ @í( $\omega \mu \varepsilon v) ;$ Paus., V, 6, $7:$ : une montagne abrupte avec des rochers escarpés ( $\pi \varepsilon ́ \tau \varrho \propto\llcorner\varsigma$

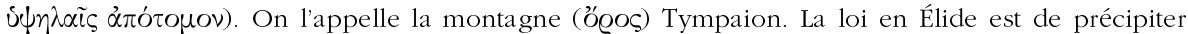

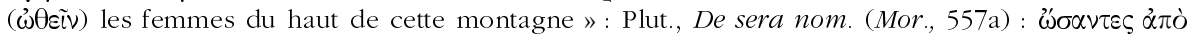

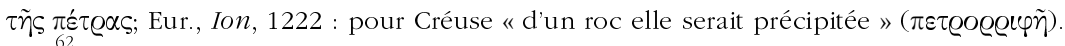

En effet l'action de précipiter ( $\pi \varepsilon \tau \varrho O Q \varrho \iota \varphi \tilde{\eta}$ [Eur., Ion, 1222] rassemble bien l'aspect pierreux 
Évidemment. Poséidon n'est pas ici un meurtrier, mais bien un « enfouisseur ». Et sans vouloir à tout prix gommer la violence de cet épisode, on doit aussi remarquer que le couple Poséidon/Érechthée ne forme pas un duo où le dieu répond à la figure usuelle du meurtrier poursuivi par sa victime telle que l'a dessinée $M$. Detienne, avec son cortège de sang, de tourment et de fuite $^{63}$. Dans ce cadre, le meurtrier peut même devenir indissociable de sa victime qui prend alors sa mémoire pour l'inquiéter de l'intérieur ${ }^{64}$. Il n'y a plus alors qu'une solution : partir.

Mais, pour notre terrain acropolitain, si Euripide raconte aussi l'indissociabilité entre Poséidon et Érechthée, ce n'est en aucun cas sur le mode de l'effroi. Si Poséidon a tué Érechthée, on le retient Semnos et c'est sous ce doux titre que les citoyens vont l'invoquer au sacrifice ${ }^{65}$. Chez Euripide, la victime s'honore avec son «meurtrier» dans le meson ${ }^{66}$ de la cité. Car il s'agit bien ici d'une alliance.

Si tout le processus autour du meurtre vise à exclure ${ }^{67}$, si la peine capitale choisit d'écarter de la cité les corps des criminels dans un chasma excentré, la

et violent de ce châtiment) est décrite avec violence comme en témoignent les verbes associés à cette action : emballô, otheô et riptô, associent bien ces idées de jeter dans, de lancer, de pousser

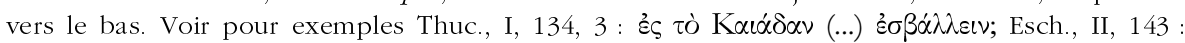

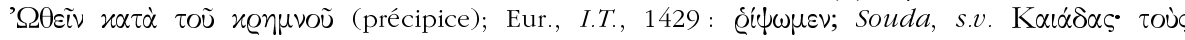

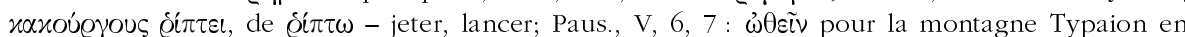

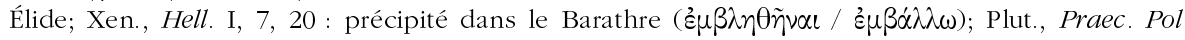

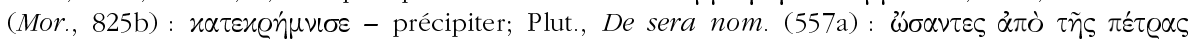
(Hyampéia Delphes); Eur., Ion, 1222: pour Créuse "d'un roc elle serait précipitée »

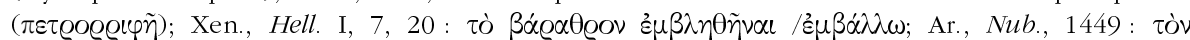

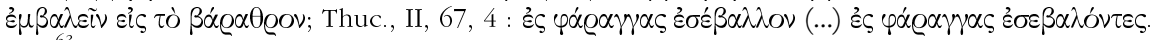

63 Voir Platon, Lois IX, 865d-e et Ant., IV, 10. Sur ces aspects, on renvoie à M. DETIENNE, "Le Doigt d'Oreste », in DETIENNE-CARTRY, o.c. (n.47), p. 29-35 et plus dernièrement Apollon le couteau à la main, o.c. (n. 1), p. 197-206. Le couple Poséidon/Érechthée n'est pas non plus similaire à celui formé par Apollon/Hyakinthos. P. Brulé les met à notre avis un peu trop en parallèle dans «Fêtes grecques: périodicité et initiations. Hyakinthies et Panathénées », in L'Initiation I, Actes du Colloque international de Montpellier, 11-14 avril 1991, Montpellier, 1992, p. 19-38. S. WIDE (Lakonische Kulte, Leipzig, 1893, p. 291 sq.) avait déjà opéré ces rapprochements entre Érechthée-Poséidon, Hyakinthos-Apollon et Néoptolème-Apollon. Or, Hyakinthos est inhumé (Paus., III, 19, 1 : $\tau \S \theta \alpha \dot{\varphi} \theta \alpha \iota$ et Pol., VIII, 28 parle de son tombeau - taphos) et surtout tué " par hasard »; et on ne peut pas trop «penser ensemble » Érichthonios et Érechthée : le tombeau dont parle P. Brulé (p. 21) est celui d'Érichthonios. Quant à Apollon, c'est vraiment le meurtrier de Néoptolème. Ici on parle d'une alliance autour de la terre.

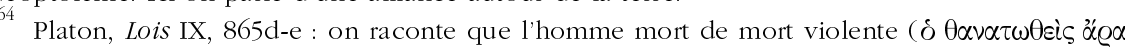

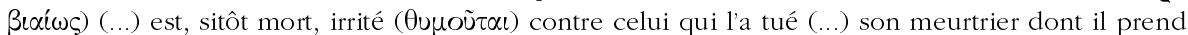

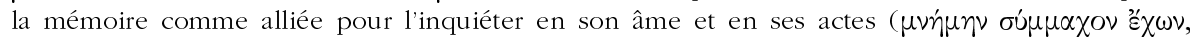

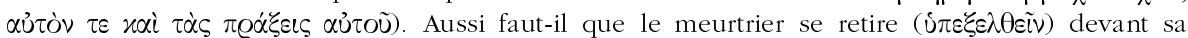
victime (...). » Voir aussi Ant., IV, 10.

${ }^{6}$ EuR., Er., 18, 92 sq. : "Érechthée prendra le nom de son meurtrier, et deviendra l'Auguste Poséidon ( $\left.\sum \varepsilon \mu \nu o ̀ \varsigma ~ \prod o \sigma \varepsilon เ \delta \tilde{\omega} \nu\right)$ : voilà comment les citoyens l'invoqueront, quand ils lui immoleront

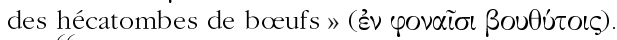

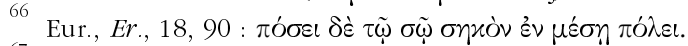

67 Purifier, c'est opérer des divisions (C. DOUGHERTY, "Murderous Founders », chapitre II in The Poetics of Colonization. From City to Text in Archaic Greece, New York / Oxford, 1993, p. 36), séparer le meurtrier, sa victime et le corps de la cité en disjoignant pollueur et territoire 
Geste d'Érechthée vise, elle, à inclure, enkyster, ramener à soi. D'un côté on fuit la terre, de l'autre on la revendique car, justement, c'est au cour de la terre que doit être enchâssé l'autochtone.

Cette lecture nous invite également à ne pas voir Érechthée comme un roi combattant dont on loue le sacrifice de sa vie. Même s'il est tombé au cours de la bataille ${ }^{68}$ sous les coups d'un Poséidon allié aux barbares Thraces ${ }^{69}$, même si les orateurs attiques ont largement utilisé cet épisode comme un écho glorieux aux très encensées Guerres Médiques ${ }^{70}$, il ne semble pas non plus croiser la catégorie de la belle mort.

\section{«Belle mort » pour l'autochtone?}

Deux vers de la tirade du messager pourraient pourtant nous inciter à le croire, car à la question pressante de la reine : " mon époux Érechthée est-il

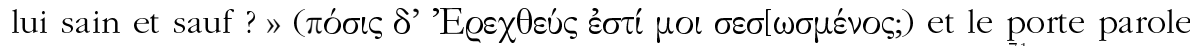
tragique répond : "il est bienheureux et fortuné tout à la fois " ( ${ }^{71}$ (x)́́@ı́c

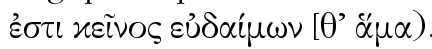

Mais, si Érechthée est «bienheureux », auréolé de l'honneur attaché à la mort au combat en Grèce ${ }^{72}$, l'Aggelos d'Euripide ne loue pas vraiment, tel un Lysias ou un Démosthène, la «belle mort» de l'autochtone. Pour Praxithéa d'ailleurs, au vers suivant, Érechthée ne peut être qualifié d'heureux que «s'il

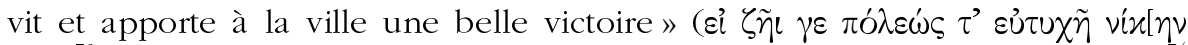
$\left.\ddot{\alpha}_{\gamma} \varepsilon\right)^{73}$. La reine éplorée se lamente ensuite en entendant la funeste nouvelle

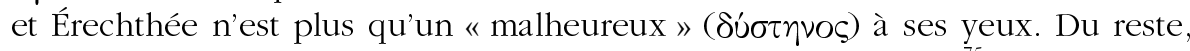
ce n'est pas sa vie qui a sauvé la cité, mais bien celle de sa fille ${ }^{75}$.

Cependant, P. Carrara et plus dernièrement C. Collard, complètent le premier travail de C. Austin ${ }^{76}$ et choisissent d'intégrer à la fin du discours du

pollué (J.-L. DuRAND, «Formules attiques du fonder » in M. DetiEnNE (éd.), Tracés de fondation, Paris / Louvain, 1990, p. 276)

68 Paus., I, 38, 3 qui raconte qu'" au cours de la bataille contre les Athéniens, Érechthée, le

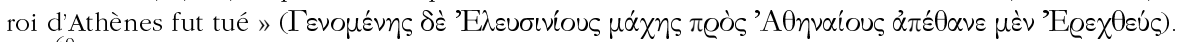

69 Pour exemples, Lyc., Leocr. 98-100; Isocr., Pan., 68.

${ }^{70}$ Pour exemples Isocr., Pan., 66 et 67, Platon, Men., 239b.

71 Eur., Er., 18, 20-21.

72 Pour makarios, voir Lys., Epit., 80. C'est surtout l'eudaimonia que l'on rencontre dans les discours des orateurs: Dem., Epit., 32 et 33; Lysias, Epit., 79 et Hyp., Epit., 42-43. Ce dernier adjectif renvoie aussi évidemment au monde des dieux.

73 Eur., Er., 18, 18.

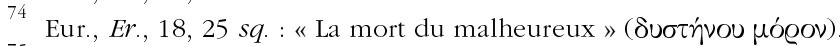

75 Eur., Er., 18, 25.

76 C. Austin, Nova Fragmenta Euripidea Euripidea in papyris reperta, Berlin, 1968, a transcrit ce fragment (établi par A. NAUcK, Tragicorum graecorum fragmenta, Hildesheilm, 1964, fr. 361) mais ne l'a pas replacé, alors que P. CARrara, o.c. (n. 9), choisit de l'insérer dans la tirade du messager (fr. 18.21-22) ainsi que C. COLlaRD (ed.), Euripides. Selected Fragmentary Plays volume 1, Warminster-England, 1997. J.C. KAMERBEEK, «Remarques sur les fragments de l'Érechthée d'Euripide », Mnemosyme 23 (1970), p. 121, avait déjà émis cette idée. 
messager deux vers perdus qui feraient alors basculer la mort d'Érechthée du côté de la kalos thanatos : "Moi, je le dis, ceux qui ont reçu une belle mort

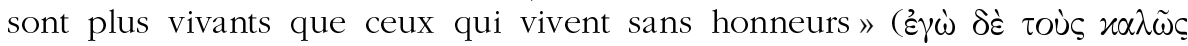

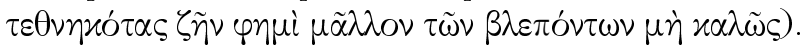

La centralité du terme $\varkappa \alpha \lambda \tilde{\omega} \varsigma^{77}$ nous rappelle cette fois plus précisément les oraisons funèbres et ce que N. Loraux a appelé "la belle mort » ${ }^{78}$. Ces vers font ainsi presque écho au texte du Ménexène qui égrène tous les avantages du "mourir» à la guerre sur le mode de la beauté $e^{80}$. D'autres orateurs ont repris ce thème, comme Lysias, qui déclame à son tour combien sont heureux les citoyens tombés au combat : ils n'auront pas à subir les affronts de la vieillesse et le déclin du corps par la maladie. Ces hommes ont, au lieu d'attendre la «mort naturelle », choisi « le plus beau des trépas », laissant ainsi un souvenir immortel ${ }^{81}$.

Mais ces vers d'Euripide sont-ils vraiment destinés à Érechthée ? Rien ne nous permet évidemment de l'affirmer. Dans une étude certes plus lointaine,

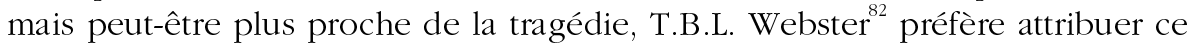
fragment à la jeune fille. C'est bien son sang qui est encensé dans les discours des orateurs et Praxithéa déclame elle-même que la mort de sa fille est le parfait écho de la mort d'un fils à la guerre ${ }^{83}$.

On peut alors se demander si ce n'est pas le sacrifice de la jeune fille qui se rapproche de la «belle mort ». Car le vrai héros de la pièce d'Euripide est féminin et pluriel, c'est la fille, c'est l'épouse d'Érechthée ${ }^{84}$. Si le roi meurt au polemos, le discours de Praxithéa que nous transmet Lycurgue est comme une oraison funèbre prononcée pour sa fille : c'est le sacrifice qui est support

77 Il faut noter l'antagonisme entre $x \alpha \lambda \tilde{\omega} \varsigma$ et $\mu \dot{\eta} \varkappa \alpha \lambda \tilde{\omega} \varsigma$ que l'on peut difficilement traduire. Sur ce terme, voir N. LORAuX, «Le Point de vue du mort », POESIE 57 (1991), p. 71.

Développé dans «La Belle mort spartiate», Ktéma 2 (1977), p. 105-120, et dans « Mourir devant Troie, tomber pour Athènes. De la gloire du héros à l'idée de la cité », SAGE 17,6 (1978), p. 801-817, ainsi que dans L'Invention d'Athènes. Histoire de l'oraison funèbre dans la 'cité classique', Paris et al., 1993, p. 120-141 surtout. Voir aussi J.-P. VERnANT, «La Belle mort et le cadavre outragé », in G. GNOLI \& J.-P. VernANT (éds), La Mort, les morts dans les sociétés anciennes, Cambridge/Paris, 1982, p. 45-75.

Platon, Men., 246d joue aussi entre $\varkappa \alpha \lambda \tilde{\omega} \varsigma$ et $\mu \grave{\eta} \varkappa \alpha \lambda \tilde{\omega} \varsigma$ : «enfants, que vos pères soient des braves, à elle seule la cérémonie présente en est la preuve : libres de vivre sans beauté, nous

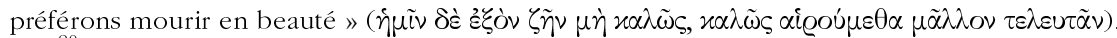

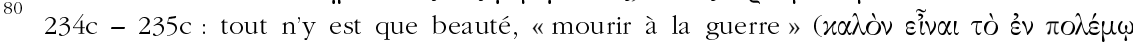

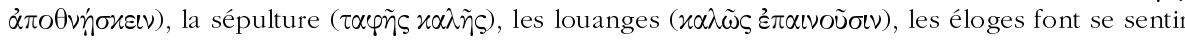

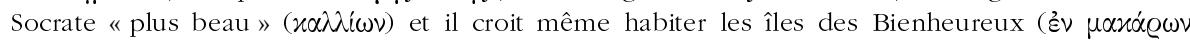

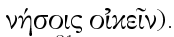

${ }^{81}$ Lysias, Epit., 79-81.

82 The Tragedies of Euripides, London, 1967, p. 129. R. AÉLION, Quelques mythes hérö̈ques dans l'ouvre d'Euripide, Paris, 1986, p. 212, indique à son tour que ce fragment « ne peut être utilisé avec certitude » et mentionne les avis divergents.

83 Lyc., Leocr., 100 : «si, au lieu de filles, des fils avaient grandi près de moi (..) ne les auraisje pas équipés de la lance pour les envoyer au combat sans redouter leur mort ? Ah! Que n'ai-je des enfants capables de combattre et de s'illustrer parmi les soldats (...). Quant à ma fille (...) seule elle mourra pour la patrie (...) n'est-il pas beau ( $\chi \alpha \dot{\lambda} \lambda$ ov) de recevoir ce privilège ?»

${ }^{84}$ Voir à ce propos le chapitre de M. DetiEnNe, « La Force des femmes », o.c. (n. 12). 
d'exemplarité et non la mort d'Érechthée ${ }^{85}$. D'ailleurs, quand Érechthée apparaît dans les épitaphioi logoi, c'est pour avoir eu le courage de donner son enfant à la patrie ${ }^{86}$. Pas son corps. Si Érechthée est mort pendant le combat, sa vie n'a pas sauvé Athènes. Il est seulement retourné vers son chthôn de naissance. En « cachette »... et sans blessure.

Ce n'est d'ailleurs pas la victoire d'Athènes qui est en jeu dans la tragédie d'Euripide : la victoire par les armes est acquise grâce au sacrifice de la jeune fille, Athènes a gagné la guerre contre le clan d'Eumolpe. Mais, cette victoire guerrière n'assure pourtant ni la stabilité, ni même la sécurité de la cité. C'est l'alliance entre Érechthée et Poséidon qui apportera la paix durable et l'ancrage recherché.

On ne réduit donc pas l'importance d'Érechthée en lui accordant seulement le statut d'un autochtone qui retourne dans son lieu de naissance. On lui reconnaît au contraire une place centrale. Enkysté par Poséidon dans la terre athénienne, le roi devient la plus sûre racine de la cité. Et c'est à deux qu'ils ont dorénavant la garde des fondations.

\section{Un autel pour deux}

C'est Athéna qui, à la fin de la tragédie, règle le sort des deux protagonistes de la pièce. Elle déclare ainsi à Praxithéa que Poséidon et Érechthée seront désormais associés dans le culte ${ }^{87}$ : «quant à ton époux, j'ordonne qu'on lui construise un sanctuaire au milieu de la ville, avec une enceinte de

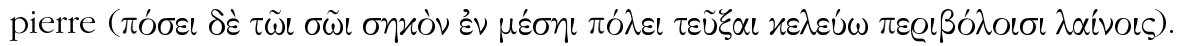
Érechthée prendra le nom de son meurtrier et deviendra l'Auguste Poséidon : voilà comment les citoyens l'invoqueront quand ils lui immoleront des

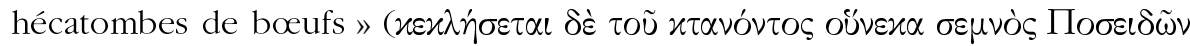

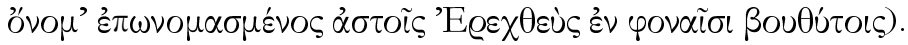

D'autres sources littéraires ou épigraphiques nous informent à leur tour de l'existence d'un culte, cette fois à Poséidon-Érechthée, comme Apollodore ${ }^{88}$, Plutarque $^{89}$ et Pausanias ${ }^{90}$. Hésychius et Athénagoras ${ }^{91}$, quant à eux, font écho à la tragédie en mentionnant l'existence d'un Érechthée-Poséidon dans la

${ }^{85}$ Lyc., Leocr., 99-100. Si on demande de l'imiter, c'est pour avoir sacrifié sa fille.

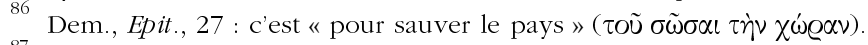

87 Eur., Er., 18.90-94. Sur le culte d'Érechthée, voir G. EKROTH, The Sacrificial Rituals of Greek Hero-Cults, Liège, 2002 (Kernos, suppl. 12), p. 175 sq. et 188 sq.

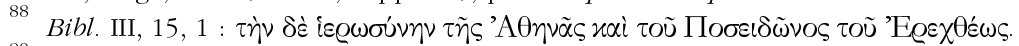

89 Vit. X Orat. (Mor., 843b-c) : "Médéios qui exerça le sacerdoce de Poséidon-Érechthée »

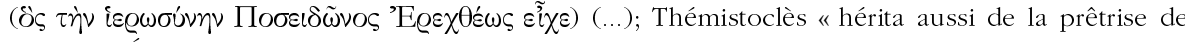
Poséidon-Érechthée ».

90 I, 26, 5 : «près de l'entrée, il y a des autels : l'un à Poséidon, sur lequel on sacrifie aussi à

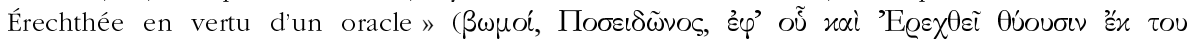
$\mu \alpha \nu \tau \varepsilon \dot{\mu} \mu \alpha \tau O \varsigma)$.

Lois 1 (Marcovich) : ici, remarquons l'inversion, "l'Athénien sacrifie à Érechthée en tant

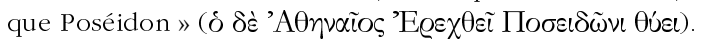


cité $^{-92}$. Les sources épigraphiques s'avèrent elles aussi plus tardives que la tragédie, exceptée une inscription du $\mathrm{v}^{\mathrm{e}}$ siècle avant J.-C. ${ }^{93}$, et ne mentionnent pas toujours Poséidon-Érechthée ${ }^{94}$ mais plus souvent Poséidon et Érechthée ${ }^{95}$.

C'est ce fameux zoi qui a exagérément focalisé l'intérêt des commentateurs qui ont en effet, tour à tour, abusivement « séparé » Poséidon et Érechthée ou au contraire trop rapproché les deux partenaires de culte ${ }^{96}$. Bien sûr, Poséidon-Érechthée n'équivaut pas à Poséidon et Érechthée mais, de toute manière, avec ou sans xaĺ, nous ne sommes pas tentés de voir dans ces deux associés une seule figure cultuelle mais bien un couple ${ }^{97}$. Ce qui est important, c'est que tous deux reçoivent ensemble des sacrifices alors qu'ils étaient séparés et adversaires dans le polemos. C'est la communauté sacrificielle qui est parlante, car c'est le signe de leur partenariat pour garantir les fondations de la cité.

Tout a été dit pourtant sur Poséidon et Érechthée : on a ainsi parlé d'identification $^{98}$, de «tentative syncrétiste », de "fusion » ${ }^{99}$ ou même d'assimilation $^{100}$. Et, à l'inverse, de "dissociation »" ${ }^{101}$. Or, Érechthée, même quand il devient Poséidon-Érechthée, n'est ni gommé, ni oublié et certainement pas «le plus faible des deux » ${ }^{102}$. Il n'est nul besoin de dire non plus qu'Érechthée était la figure divine la plus ancienne, présent bien avant que Poséidon ne le

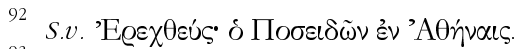

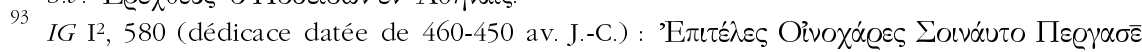

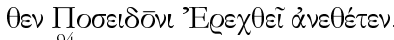

Comme $I G \mathrm{II}^{2}, 4071,1.25-27$, base de marbre trouvée à Éleusis (milieu du II $^{\mathrm{e}}$ siècle av. J.-

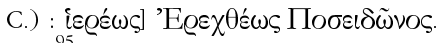

C'est le cas de $I G \mathrm{II}^{2}, 1146$ ( $L S C G 31=$ SEG XXV, 140) : ce décret de tribu trouvé sur

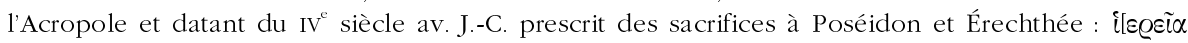

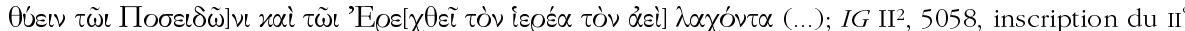
siècle av. J.-C. qui se trouve sur le siège du prêtre de Poséidon et d'Érechthée au théâtre de

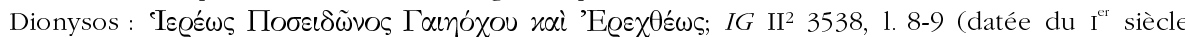

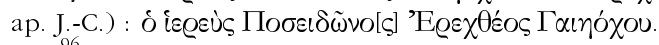

On peut toujours gloser sur l'hypothétique omission d'un xoí. Au lieu de prendre les inscriptions $I G \mathrm{II}^{2}, 3538$ et $I G \mathrm{II}^{2}, 4071$ (o.c. notes précédentes) telles qu'elles nous sont parvenues, M. LACORE ("Euripide et le culte de Poséidon-Érechthée », REA 85 [1983], p. 221), pour être sûre qu'il s'agit bien d'une 'fusion' entre Érechthée et Poséidon, a besoin d'attester que sur ces inscriptions au moins, «l'ordre des mots interdit l'hypothèse d'un xá omis ».

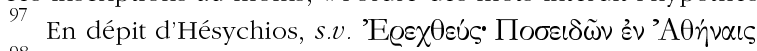

98 Pour exemples, voir J.D. MIKALSON, "Erechtheus and The Panathenaia », AJPh 97 (1976), p. 143; C. Austin, "De nouveaux fragments de l'Érechthée d'Euripide », Recherches de Papyrologie 4 (1969), p. 59; COLLARD, o.c. (n. 76), p. 193 (lui nuance : ce culte «suggest a cultic identification »).

LACORE, o.c. (n. 96) qui utilise plus dernièrement l'expression «Érechthée fondu avec son divin meurtrier», in o.c. (n. 16), p. 89. Pour la "fusion », voir aussi F. JouAn, " Héros tragique et deus ex machina chez Euripide », in Kernos, suppl. 10 (2000), p. 35

100 W. BuRKerT, Homo Necans. The Anthropology of Ancient Greek Sacrificial Ritual and Myth, Berkeley et al., 1983, p. 149 : « two names for a single god » et Brulé, o.c. (n. 63), p. 22.

101 LACORE, O.c. (n. 96), p. 218

102 Comme le dit LACORE, o.c. (n. 96), p. 222 
supplante ${ }^{103}$. Le temple qui abrite leur autel commun porte d'ailleurs le nom de l'autochtone - l'Érechthéion, et Hésychius, comme Athénagoras, rappellent que c'est Poséidon qui porte le nom d'Érechthée dans la cité ${ }^{104}$. Il faut plutôt remarquer que, même quand Poséidon et Érechthée sont séparés par le xaí, ils peuvent avoir le même prêtre ${ }^{105}$. N'est-ce pas cela le plus symbolique? C'est pourquoi nous préférons parler d'association, de collaboration et de complémentarité.

L'inscription la plus révélatrice pour comprendre ce partenariat fondateur est sûrement celle qui mentionne Poséidon Gaièochos Érechthée ${ }^{106}$ : elle dit combien Érechthée et ce Poséidon Qui tient la terre sont liés par la terre, autour de la terre et pour la terre, qui est d'ailleurs le véritable enjeu de la tragédie $^{107}$. Sans Poséidon, à qui il est attaché dans le culte, Érechthée n'est qu'un « autochtone de plus », une énième racine. Et sans Érechthée, Poséidon n'intègre pas le cercle très fermé des acteurs de la (des) fondation(s) de la cité, car cet autel commun avec l'autochtone est vraiment la reconnaissance de Poséidon comme fondateur. Un fondateur à l'assise sûre et aux fondations inébranlables.

En dépit des divergences concernant le complexe dossier archéologique de l'Érechthéion ${ }^{108}$, le voisinage demeure bien le même et il ne saurait être remis en cause : c'est d'ailleurs ce qui fait de cet ensemble inédit le lieu de mémoire des origines qui sont, on le voit, plurielles. Oui, les Athéniens sont les enfants des dieux, nés de la terre civique, autochtones, installés depuis toujours et pour toujours. Mais cette belle naissance, cet ancrage déclaré immémorial, il faut le redire, il faut renouer le lien à la terre. À Héphaïstos

103 C'est la thèse de Burkert, o.c. (n. 100), p. 169 et de F. SCHACHERMEYR, Poseidon und die Entstehung des griechischen Götterglaubens, Bern, 1950, p. 11. On ne peut être aussi affirmatif, je crois. De même, les questions de chronologie s'avèrent fort complexes et on ne saurait démêler quand s'est fixé ce double culte. LACORE, o.c. (n. 96), p. 218, a recensé les différents points de vue. Elle échafaude une théorie qui nous apparait bien fragile : en effet, pour elle, l'inscription $I G$ $\mathrm{II}^{2}, 5058$ du II ${ }^{e}$ siècle av. J.-C. (cité supra note 95) est charnière « puisque c'est après elle que l'on voit les témoins de la fusion l'emporter sur ceux de la dissociation de Poséidon et d'Érechthée ». MikAlson, o.c. (n. 98), p. 143 sq. voit lui dans cette mention d'Euripide la preuve de l'existence du culte au v viècle av. J.-C., mais il est un peu seul. Certains parlent d'une innovation de la part d'Euripide ou pensent que ces vers font écho à la construction d'un nouveau temple qui serait l'Érechthéion comme l'évoque Brulé, o.c. (n. 63), p. 26.

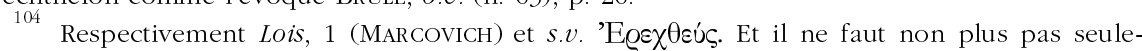
ment retenir comme LACORE (o.c. [n. 16], p. 90) dans cet ordre d'Athéna une « quadruple apothéose », celle des trois jeunes filles et celle du roi, mais une alliance.

${ }^{105} I G \mathrm{II}^{2}, 1146$ et $I G \mathrm{II}^{2}, 5058$ (citées note 95).

$106 I G \mathrm{II}^{2}, 5058$ (siège du prêtre de Poséidon Gaièochos et Érechthée au théatre de Dionysos, II ${ }^{\mathrm{e}}$ s. av. J.-C.); $I G \mathrm{II}^{2} 3538$ (prêtre de Poséidon Érechthée Gaièochos, I ${ }^{\text {er }}$ s. ap. J.-C.).

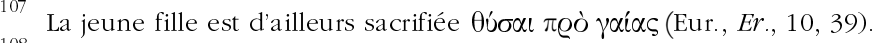

108 Sur l'Érechthéion, sa localisation et les sacrifices qui y étaient rendus, voir K. JEPPESEN, "Where was The So-Called Erechtheion ", AJA 83 (1979), p. 381-394 et The Theory of The Alternative Erechtheion, Aarhus, 1987; LACORE, o.c. (n. 96) et P. Brulé, "La Cité en ses composantes : remarques sur les sacrifices et la procession des Panathénées », Kernos 9 (1996), p. 37-63. 
l'ascendance divine ${ }^{109}$, à Érechthée l'autochthonie, à Athéna le titre de Poliade et à Poséidon l'ancrage sur la terre athénienne. Si Héphaïstos est le "géniteur », Poséidon est le fondateur. Chacun est indispensable et partenaire de l'autre puisqu'Athéna seule ne garantit pas la fondation de la cité. Il a fallu que Poséidon intègre ce cercle pour tenir-soutenir les fragiles premières fondations d'Athènes.

\section{D'Érichthonios à Érechthée, d'Athéna à Poséidon}

Le rôle de Poséidon s'avère bien le symétrique de celui d'Athéna dans la naissance d'Érichthonios : si la Poliade a le premier rôle (comme toujours), en recevant dans ses bras le premier autochtone, Poséidon assume le rôle de la consolidation. Et ce n'est pas le moindre, car c'est lui qui redit et pérennise le lien indéfectible que les Athéniens ont déclaré avoir avec leur terre. C'est donc à deux qu'ils se partagent l'autofondation et la refondation de la cité. Quelques vers d'Euripide les mettent parfaitement en écho, chacun à un bout de la chaîne autochtone : deux épisodes, naissance d'Érichthonios et enfouissement d'Érechthée, qui semblent d'ailleurs résumer toute l'histoire de la cité, car ce sont les deux questions qu'Ion pose à Créuse sur Athènes ${ }^{110}$ :

Créuse : Le lieu de ma naissance est la ville d'Athènes

Ion : $\quad$ Illustre est ta cité (...) c'est donc vrai ce que disent les gens (...) Ton

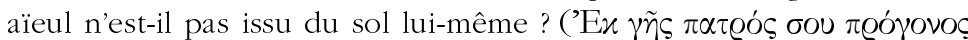

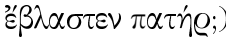

Créuse : Érichthonios ? Oui (...)

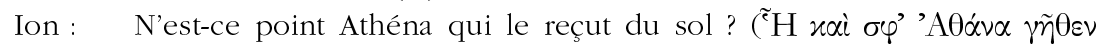

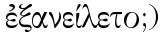

Créuse : Oui, dans ses bras de vierge (...)

Ion : $\quad$ Ah ! Et cette autre histoire (...) Ton père fut vraiment englouti par le

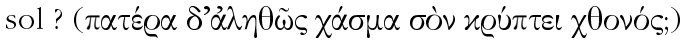

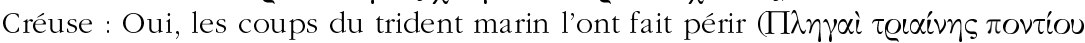
$\left.\sigma \varphi^{\prime} \dot{\alpha} \pi \dot{\omega} \lambda \varepsilon \sigma \alpha \nu\right)$.

Érichthonios naît de la terre, Érechthée meurt dans la terre, tels sont leurs sorts. À leurs côtés, les deux dieux fondateurs de la cité Athéna et Poséidon se font écho et redoublent les liens avec le territoire. On se partage bien à Athènes entre Poséidon et Athéna les autochtones et donc les opérations de fondation.

Érechthée englouti dans la terre athénienne rappelle ainsi parfaitement la première «cachette », celle du sperme d'Héphaïstos, elle-même déclinée sur le mode du kruptôn ${ }^{111}$. Érechthée raconte parfaitement l'histoire athénienne:

109 Il est le "père » des Athéniens (ne l'oublions pas trop), Esch., Eum., 13 : « les enfants

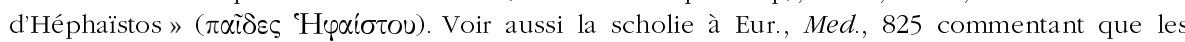

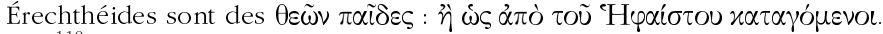

${ }^{110}$ Eur., Ion, 262-282

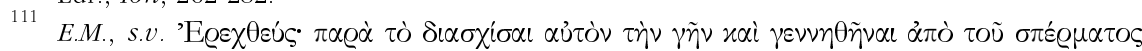


une histoire de la terre à la terre, une histoire de naissance qui devient ancrage.

Avant, Athènes ne faisait que multiplier des naissances autochtones : c'est Érechthée qui, grâce à Poséidon, clôture la longue chaîne des opérations de fondation. En cela, il n'est pas qu'un «maillon important » ${ }^{112}$ de la chaîne autochtone, mais l'ultime racine enfouie dans le chthôn par ces Athéniens en mal d'ancrage.

Gardien des origines, garant de la stabilité, Poséidon siège désormais avec Érechthée sur l'Acropole de la cité autochtone qui, grâce à lui, a réussi le pari de son autofondation. Car, à travers Érechthée, c'est tous les Athéniens que Poséidon enracine sur leur propre terre.

SOnia DARTHOU

Centre Louis Gernet

9, rue Vivienne

$\mathrm{F}-75002$ PARIS

Courriel : soniadarthou@yaboo:fr

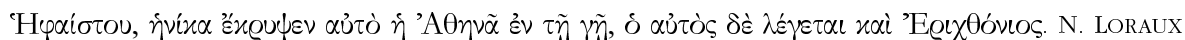
«Mourir devant Troie », o.c. (n. 78), p. 35-36, va encore plus loin, éclairant la pratique funéraire avec le postulat d'autochthonie; confier les morts à la terre c'est alors ainsi «assurer la reproduction de la cité ». Elle commente notamment Platon, Men., 237c.

12 N. LoRauX, Les Enfants d'Athéna. Idées athéniennes sur la citoyenneté et la division des sexes, Paris, 1990, p. 38. 